\title{
Multi-technique study of the anodic degradation of polyaniline films ${ }^{1}$
}

\author{
Huyen N. Dinh, Jianfu Ding, S.J. Xia, Viola I. Birss* \\ Department of Chemistry, University of Calgary, Calgary, Alberta, T2N 1N4, Canada
}

Received 25 November 1997; received in revised form 15 June 1998

\begin{abstract}
Polyaniline (PANI) films, formed by potential cycling methods on Au electrodes in sulfuric acid solutions, were anodically degraded with time at ca. $1 \mathrm{~V}$ versus RHE. Ellipsometric and cyclic voltammetric data suggest that colored species are formed within the film, perhaps containing quinone endgroups, and the bulk of the film dissolves uniformly creating greater porosity. This model of film degradation has been supported by transmission electron microscopy (TEM) studies of cross-sectional samples of PANI films. Both TEM and ellipsometry also showed that the PANI film is not delaminated, and is very resistant to anodic dissolution/degradation. Concurrent in situ mass measurements suggest that, in the early stages of film degradation, some regions of the film become electrochemically inactive, although the nature of the charge compensation mechanism of the PANI redox reaction remains unaltered. When no further film degradation can be accomplished, the mass data show that a substantial quantity of largely inactive film material is still present on the electrode surface. Furthermore, the change in the redox potential and the invariant charge compensation stoichiometry suggests that, although the final degraded film is a different material from the newly formed PANI film, only those portions of the film that have PANI characteristics are electrochemically active. (C) 1998 Elsevier Science S.A. All rights reserved.
\end{abstract}

Keywords: Polyaniline; Ellipsometry; Quartz crystal microbalance; Transmission electron microscopy; Degradation; Stability

\section{Introduction}

Considering the many practical applications of polyaniline (PANI) films, the retention of their excellent capacitive (up to $0.15 \mathrm{~F} \mathrm{mg}^{-1}$ [1]) and kinetic (e.g. 100 $\mu$ s to switch between conducting and non-conducting states [2]) characteristics, as well as their physical and chemical stability, with time, is of utmost importance. Clearly, the loss of film material and/or of electrical contact to segments of the film would pose a problem for PANI films used in battery, supercapacitor, electrochromic, etc. applications [3-8]. Also, the loss of film stability would be a major problem when PANI films are employed as corrosion protection coatings [9-14].

\footnotetext{
* Corresponding author. Tel.: +1 403 2206432; fax: + 1403 2899488; e-mail: birss@,acs.ucalgary.ca

${ }^{1}$ Presented at the Electrochemical Society Symposium: Processes in Polymers and Polymer|Metal Interfaces, Montreal, 4-9 May 1997.
}

It is well known that, particularly in acidic solutions, PANI films are susceptible to dissolution processes at potentials greater than ca. $0.9 \mathrm{~V}$ versus RHE [15-24]. The principal product appears to be soluble benzoquinone (BQ) $[15,16,18,20,30]$, which has been detected in solution by ring-disc studies [20,30], spectrophotometry $[15,20,22]$, and by comparison of the new cyclic voltammetric peak $[22,25]$, which appears at ca. $0.75 \mathrm{~V}$ versus RHE, with the redox peaks of the benzoquinone/hydroquinone (BQ/HQ) couple when it is added intentionally to solution. Other insoluble degradation products have been suggested to remain on the electrode surface, including PANI strands containing quinoneimine end groups $[16,20]$ and ortho-coupled polymers [22].

The goal of the present paper is to examine the nature of the PANI degradation process in more detail, primarily to establish the structural/morphological and electrochemical properties of the PANI film as a func- 
tion of the extent of film degradation. It is also of interest to determine whether the PANI $\mid$ metal interfacial region is altered significantly during this process, in order to determine how much the corrosion protection properties of the PANI film are compromised with time. The properties of the film, once it becomes resistant to further degradation, are also examined here. All PANI films studied in the present work were formed on polycrystalline $\mathrm{Au}$ surfaces in 1 mol $1^{-1}$ sulfuric acid by the potential cycling method. Films up to $160 \mathrm{~nm}$ in thickness were examined, and film degradation was achieved by holding at potentials of ca. $1 \mathrm{~V}$ versus RHE for various periods of time. The film properties were examined simultaneously by ellipsometry, quartz crystal microbalance techniques and cyclic voltammetry as a function of the extent of degradation. These results obtained for PANI films at various stages of degradation have been confirmed by cross-sectional TEM analyses.

\section{Experimental}

\subsection{Equipment}

Cyclic voltammetry (CV) was performed using EG\&G PARC 173/175 instrumentation, while the quartz crystal microbalance (QCMB) experiments were carried out using a home-made oscillator, connected to a Philips PM 6654C programmable high resolution frequency counter. All electrochemical and frequency data were recorded using a BBC SE 790 and SE $780 \mathrm{X} / \mathrm{Y}$ recorder, respectively. A Gaertner L116C ellipsometor was used in the monochromatic mode to monitor the optical properties of the PANI film at ca. $0 \mathrm{~V}$ versus RHE. The light source employed was a $\mathrm{He}-\mathrm{Ne}$ laser at a wavelength of 632.8 $\mathrm{nm}$. The angle of incidence employed for all measurements was $70^{\circ}$ and the polarizer angle was $45^{\circ}$. The instrumentation and all ellipsometric data collection were controlled by an IBM computer, interfaced to the system. The refractive index of $1 \mathrm{~mol} \mathrm{l}^{-1}$ sulfuric acid solution, as measured with a refractometer, was 1.36. Simulation of the experimental data, $\Psi$ and $\Delta$, was carried out by assuming a homogeneous film model, using a routine described elsewhere [26].

A Hitachi H-7000 transmission electron microscope (TEM) was employed to examine the cross-sectional nanostructure and film thickness of the PANI film. All TEM specimens examined were prepared on a Reichert-Jung E microtome.

\subsection{Electrodes and cells}

An electrochemical/optical cell, constructed of
Plexiglass, was used for concurrent CV, QCMB, and ellipsometry experiments. The working electrode (WE) was an AT-cut quartz crystal (Valpey-Fisher, $5 \mathrm{MHz}, 2.5 \mathrm{~cm}$ diameter), sputter coated first with a thin film of titanium for adhesion and then a thin film of gold. The crystal constant was $56.6 \mathrm{~Hz} \mathrm{~cm}^{2}$ $\mu \mathrm{g}^{-1}$, according to the Sauerbrey equation [27]. The resonant frequency of the oscillating PANI-coated quartz crystal was measured in situ as a function of potential. The gold-sputtered quartz crystal WE, sandwiched between two O-rings, entered the cell through its base. The two optical quartz crystals, used for entry and exit of the $\mathrm{He}-\mathrm{Ne}$ light source, were situated on opposite sides of the cell (angle between cell walls and cell base was $70^{\circ}$ ). A large area Pt gauze counter electrode (CE), a reversible hydrogen reference electrode (RHE), and a $\mathrm{N}_{2}$ gas bubbler were introduced into the cell via ports on the side of the cell. All potentials in this paper are reported versus the RHE and current and charge densities are given with respect to the apparent electrode area $\left(0.45 \mathrm{~cm}^{2}\right)$. Ellipsometric, QCMB and $\mathrm{CV}$ responses were recorded simultaneously using a sweep rate of $50 \mathrm{mV} \mathrm{s}^{-1}$.

Standard three-electrode, two compartment glass cells were used to prepare PANI specimens electrochemically for TEM studies. A polycrystalline gold foil (Aldrich, 99.9+\%, $0.025 \mathrm{~mm}$ diameter; AESAR, $99.99 \%, 0.1 \mathrm{~mm}$ diameter), embedded in a Teflon holder, was used as the WE. After the gold foil had been electrochemically coated with PANI, a thin layer of copper was first sputtered on its surface in order to enhance the contrast between the PANI film and the Formvar resin coated $\mathrm{Cu}$ grids (JBS Super200) as seen under the TEM, and then embedded in Spurr's resin (provided as a kit sold by Ernest F. Fullam) in a silicon rubber mold. The resin, containing the sample, was then cured at $60^{\circ} \mathrm{C}$ for a minimum of $8 \mathrm{~h}$. The sample block was then trimmed and shaped, using an ultramicrotome, to a pyramid shape having a ca. $0.1 \mathrm{~mm}^{2}$ trapezoidal tip area. A glass or diamond knife was used to prepare 30-50 $\mathrm{nm}$ thick cross-sections, which were collected on the $\mathrm{Cu}$ grids, and then observed under the TEM.

\subsection{Solutions and general experimental conditions}

Sulfuric acid (BDH, ACS Assured, 95.0-98.0\% assay) was used as received. Aniline (Fischer Scientific, Certified ACS) was distilled prior to use. All solutions were prepared with triply distilled water. All experiments were carried out at room temperature. Solutions were de-aerated by purging nitrogen either through or above the cell solution during all of the experiments. 


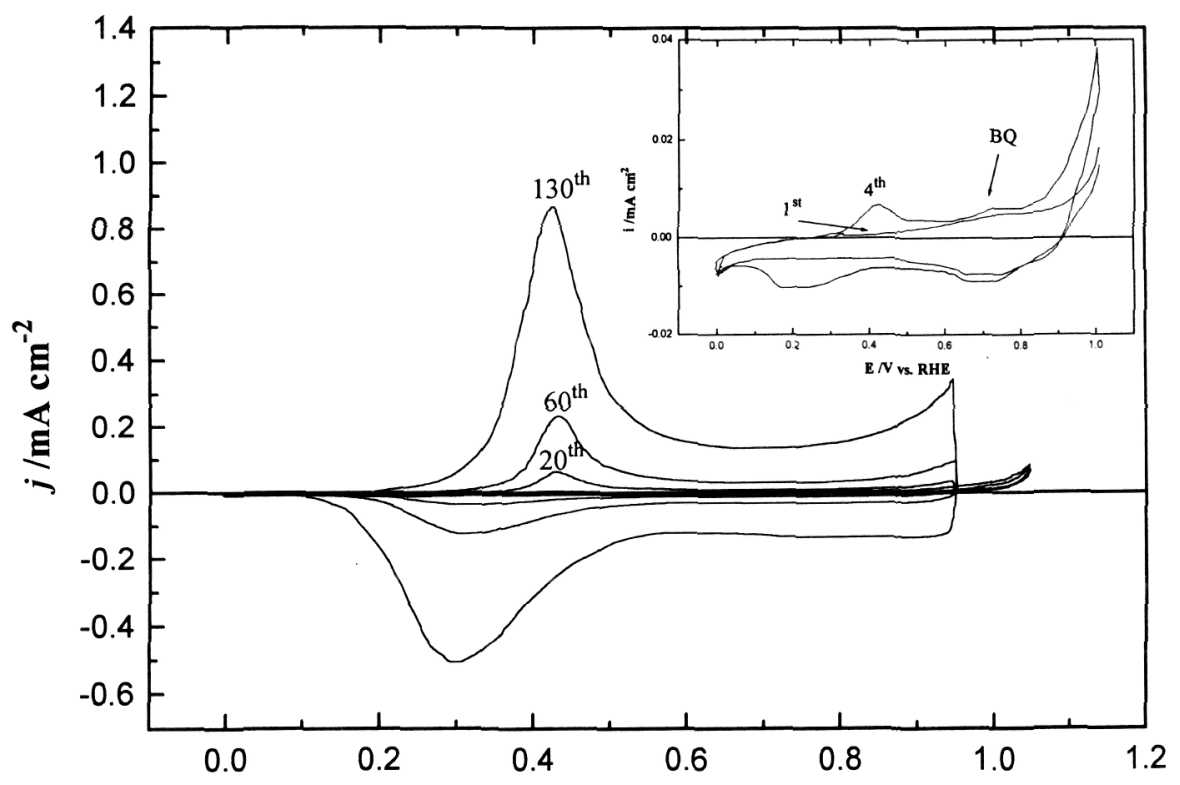

(a)

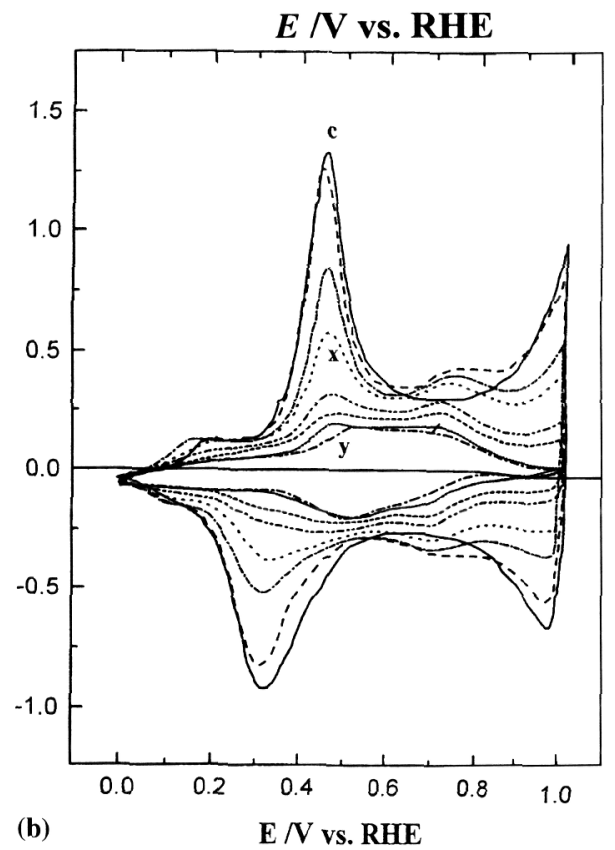

Fig. 1. (a) Typical $\mathrm{CV}$ responses $\left(50 \mathrm{mV} \mathrm{s}^{-1}\right)$ during PANI growth in $0.1 \mathrm{~mol}^{-1}$ aniline $+1 \mathrm{~mol}^{-1} \mathrm{H}_{2} \mathrm{SO}_{4}$, deposited on a Au $\mid$ quartz crystal substrate. The 20th, 60th and 130th cycles are shown, involving scanning between 0 and $0.95 \mathrm{~V}$. Inset: the first to fourth cycles involved scanning between 0 and $1.05 \mathrm{~V}$. (b) Typical CVs of PANI in $1 \mathrm{~mol} \mathrm{l}^{-1} \mathrm{H}_{2} \mathrm{SO}_{4}$, after various times of holding at $1 \mathrm{~V}: 0(-), 1 \mathrm{~min}(--), 4 \mathrm{~min}(-\cdot--)$, $6 \min (\ldots), 12 \min (-\cdots), 17 \min (--), 27 \min (\cdots)$ and $32 \min (-\cdot)$. Labels $\mathbf{c}, \mathbf{x}$ and $\mathbf{y}$ are cross-referenced to Fig. 3, indicating various stages of PANI degradation.

\section{Results and discussion}

\subsection{CV response during PANI growth versus during anodic degradation}

Fig. 1 (a) shows a series of CVs during PANI film growth on a gold-sputtered quartz crystal surface. The film was formed by cycling at $50 \mathrm{mV} \mathrm{s}^{-1}$ between 0.00 and $1.05 \mathrm{~V}$ versus RHE for the first three to five cycles and then between 0.00 and $+0.95 \mathrm{~V}$ for subsequent cycles in a $0.1 \mathrm{~mol} \mathrm{l}^{-1}$ aniline $+1 \mathrm{~mol} \mathrm{l}^{-1} \mathrm{H}_{2} \mathrm{SO}_{4}$ solution. It can be seen (see inset in Fig. 1 (a)) that, in the first few cycles of potential, a pronounced anodic peak at $0.75-0.80 \mathrm{~V}$, thought to reflect the $\mathrm{BQ} / \mathrm{HQ}$ redox process $[15,16,20,22]$, is present, while in subsequent cycles, this peak does not increase further in size and the main PANI redox peaks, centered at $0.43 \mathrm{~V}$, begin to dominate the $\mathrm{CV}$ response. The thickness of the PANI film in the cycles shown in Fig. 1 (a) has been estimated based on TEM cross-sectional measurements 
(see below). A final film thickness of $160 \mathrm{~nm}$ was achieved after ca. $2 \mathrm{~h}$ of cycling, i.e. after 190 cycles of potential. The CV charge for the 190th cycle, determined between 0 and $0.95 \mathrm{~V}$ in aniline + sulfuric acid solution, is $7.25 \mathrm{mC} \mathrm{cm}^{-2}$.

Fig. 1 (b) shows a typical set of CVs after various times of anodic degradation of the PANI film by holding at $1.0 \mathrm{~V}$. It should be noted that the $\mathrm{BQ} / \mathrm{HQ}$ redox peaks, centered at $0.75-0.80 \mathrm{~V}$, become quite prominent after the first minute of holding at $1 \mathrm{~V}$ and remain relatively constant in size until after 3 min of holding at $1.0 \mathrm{~V}$. In contrast, the principal PANI redox peaks decrease rapidly at early times, and then become more stable at longer times of degradation. These results are shown more clearly in Fig. 2, in which the anodic CV charge in the main PANI redox peaks $(0-0.6 \mathrm{~V})$, the anodic charge in the region of the $\mathrm{BQ} / \mathrm{HQ}$ redox peaks $(0.6-0.9 \mathrm{~V})$, and the overall anodic charge $(0 \sim 0.9 \mathrm{~V})$ are shown as a function of time at $1.0 \mathrm{~V}$. The charge passed during the time of holding at $1 \mathrm{~V}$ was not measured. It is not certain how the anodic charge between 0.6 and $0.9 \mathrm{~V}$ should be determined. The data in Fig. 2 did not involve extrapolation of the current defining the PANI peaks to estimate a baseline. Rather, a baseline of zero current for the BQ peak has been assumed, as has been done also in the past literature. In a similar approach, the ratio of the PANI redox peak current to that of the BQ peak has been used in prior work $[17,22,25]$ to estimate the extent of PANI degradation as a function of film thickness and growth solution employed.

Fig. 1 (b) also shows that, after ca. $30 \mathrm{~min}$ at $1.0 \mathrm{~V}$, as further changes in the $\mathrm{CV}$ become more difficult to induce, the PANI redox peak begin to move positively by ca. $0.1 \mathrm{~V}$, while the $\mathrm{BQ}$ degradation peaks move negatively, becoming centered at $0.68 \mathrm{~V}$. After still longer times at $1 \mathrm{~V}$, the two peaks appear to merge

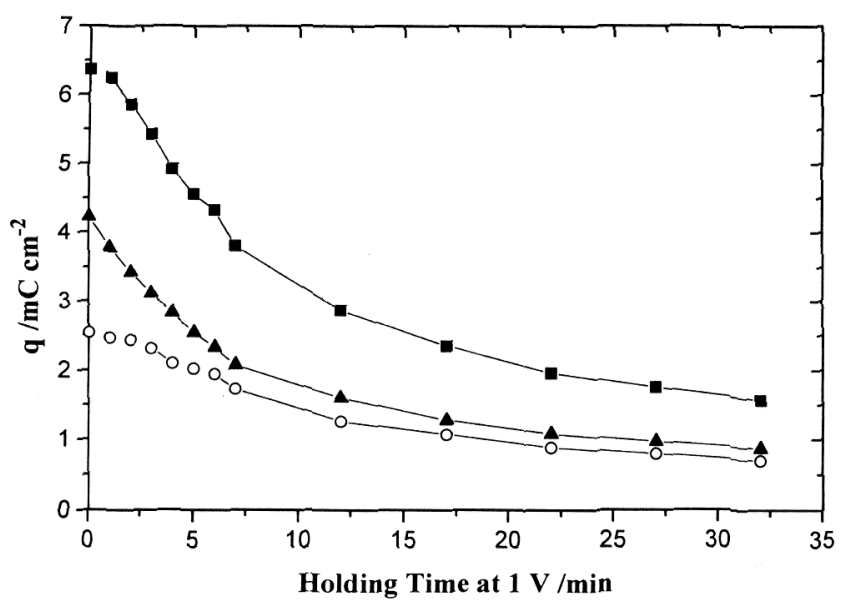

Fig. 2. Anodic charge density over various potential ranges versus the total time of holding at $1 \mathrm{~V} ; 0-0.9 \mathrm{~V}(\mathbf{\square}), 0-0.6 \mathrm{~V}(\mathbf{\Delta})$, and $0.6-0.9$ $\checkmark(0)$.

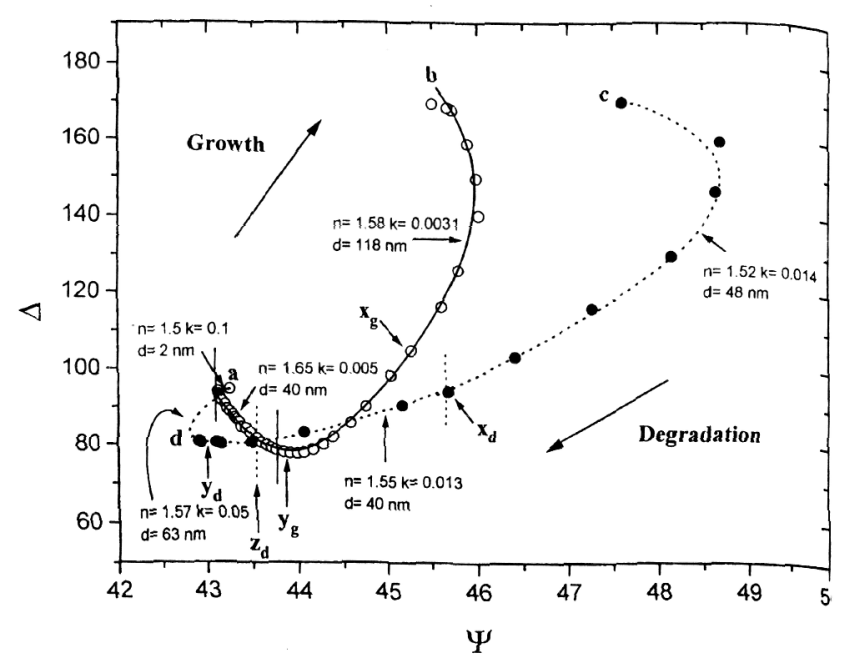

Fig. 3. Best fit $\Delta$ versus $\Psi$ curves for a $160 \mathrm{~nm}$ PANI film during growth (-) and degradation (---). Segment a-b was obtained during the growth of PANI in aniline + sulfuric acid solution, starting with the bare gold substrate (a) until after 190 cycles of PANI growth (b). Curve c-d represents the data obtained during the degradation of PANI film, from when it was first transferred to fresh sulfuric acid solution (c) after storage in air overnight, to after ca. $40 \mathrm{~min}$ of holding at $1 \mathrm{~V}$ (d). Circles represent the experimental ellipsometric data gathered at $0 \mathrm{~V}$ during growth and degradation. The parameters $n, k$, and $d$ used to fit the ellipsometric data are indicated at various points on both the growth and degradation curves. The vertical lines separate the film layers of different properties on both the growth $(-)$ and degradation (--) curves. Labels $\mathbf{x}$ and $\mathbf{y}$ indicate the various stages of growth (subscript $\mathbf{g}$ ) and degradation (subscript $\mathbf{d}$ ) where the film has similar thicknesses.

together, indicating that the properties of the remaining film are different from those of the original PANI film. These results may, therefore, suggest the development of a new polymer phase, perhaps one having a PANI skeleton interspersed by quinoneimine groups or a cross-linked polymer consisting of PANI and benzoquinone. The fact that the current at $1.0 \mathrm{~V}$ is now very small suggests that this new polymer phase consists primarily of highly oxidized functional groups.

\subsection{Ellipsometric and TEM study of PANI film degradation}

\subsubsection{PANI film growth}

The ellipsometry data, gathered at $632.8 \mathrm{~nm}$ during the growth and degradation of the PANI film of Fig. 1, are shown in Fig. 3. Each period of film growth was followed by a potential scan at $50 \mathrm{mV} \mathrm{s}^{-1}$ to $0.0 \mathrm{~V}$, where the ellipsometric data were gathered in the reduced, transparent form of PANI. Then the potential was scanned positively again to grow more film, up to a final film thickness of $160 \mathrm{~nm}$. Film degradation was achieved by holding the potential at $1.0 \mathrm{~V}$ for $1 \mathrm{~min}$ periods, and later for $5 \mathrm{~min}$. After each period of degradation, the potential was scanned between 0.0 and $0.6 \mathrm{~V}$ until a steady-state $C V$ was obtained, and then 
the ellipsometric data were collected at $0.0 \mathrm{~V}$ for direct comparison with the film properties during growth. The hysteresis between the two sets of data (open vs. closed circle symbols in Fig. 3) suggests that the PANI film is quite different in the two cases, for films of the same film thickness (examples indicated by the $\mathbf{x}$ and $\mathbf{y}$ markers, with the subscript of $\mathbf{g}$ and $\mathbf{d}$ indicating the PANI film thickness during growth and degradation, respectively), consistent with that reported by Greef et al. [28] and with the CV results of Fig. 1 (a versus b).

The model which best fits the growth data is as follows. Initially, a very thin layer of film, up to $2 \mathrm{~nm}$ thick, and having a refractive index, $n$, of 1.5 and an extinction coefficient, $k$, of 0.1 , is deposited. This relatively high $k$ value will be shown below to be typical of the condition when the $\mathrm{BQ} / \mathrm{HQ}$ redox peaks are dominant in the CV. Notably, the completion of this stage of film growth correlates with cycle no. 3 in the CVs of Fig. 1 (a), after which the PANI redox peaks increase in size at a much more rapid rate than do the $B Q / H Q$ redox peaks. As further film growth occurs with subsequent potential cycling, a uniform and compact film, with a higher $n$ and lower $k$ value $(n=1.65, k=0.005)$, is formed above the $2 \mathrm{~nm}$ inner film. The final ellipsometrically calculated thickness of this compact layer is ca. $40 \mathrm{~nm}$. Further growth results in the formation of a uniform, but more porous, PANI film $(n=1.58, k=$ 0.0031 ) on top of the compact layer. These $n$ values compare favorably with the $n$ values of 1.48 to 1.6 , obtained by Gottesfeld et al [29-33], for the reduced form of PANI, grown using constant current growth methods in $\mathrm{HCl}$ solutions, at least for films up to ca. 80 $\mathrm{nm}$ in thickness. A value of $n$ of 1.56 was obtained for reduced PANI films, grown on $\mathrm{Pt}$, in $\mathrm{HCl}$ solutions by potential cycling [28].

Fig. 4 shows a plot of the film thickness, predicted from the modeling of the ellipsometric data (Fig. 3), as

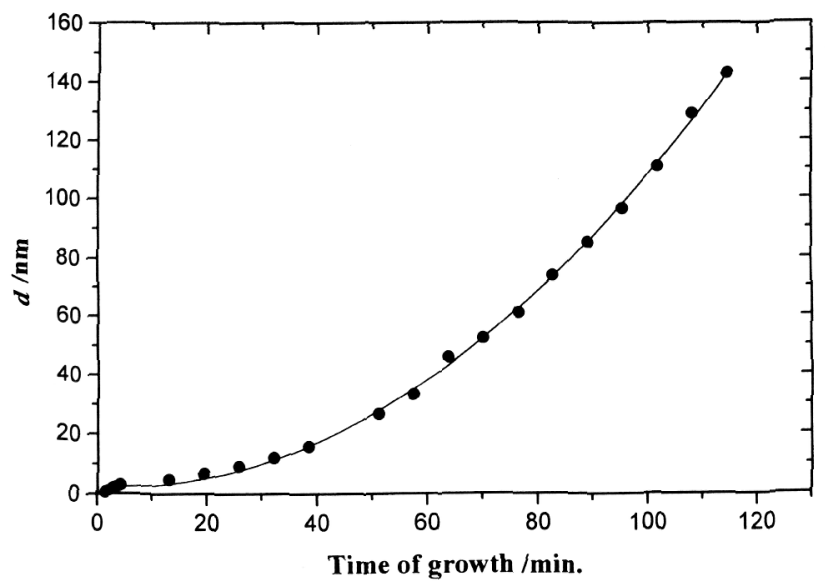

Fig. 4. Film thickness, $d$, predicted from the modeling of the ellipsometric data (Fig. 3) versus time of cycling during film growth at 50 $\mathrm{mV} \mathrm{s}^{-1}$. a function of the time of cycling at $50 \mathrm{mV} \mathrm{s}{ }^{-1}$ during film growth. The growth curve is similar in shape to that reported in the past literature [28,34]. It is seen that the initial growth rate of the film is slow, presumably during the formation of the compact inner layers. The growth rate becomes more rapid with time, as the surface area of PANI increases, consistent with the proposed autocatalytic polymerization mechanism of PANI growth [35].

The suggested uniform growth model used here to model the ellipsometric data is in agreement with the prior literature $[28,30,31,34]$, although a distinction between the growth of an inner, compact layer and an outer, more porous film has not been made in the published ellipsometric work. Further support for the growth model arising from Fig. 3 comes from the fact that the film thicknesses predicted from the model are very close to those found by parallel TEM cross-sectional analyses. Fig. 5 shows a typical example of a TEM cross-section of a PANI film, formed for the same total growth time and using other similar growth conditions to those used in the ellipsometry experiments in Fig. 3. The polymer film, sandwiched between the $\mathrm{Au}$ electrode substrate and an overlayer of $\mathrm{Cu}$, is seen to be $160 \pm 10 \mathrm{~nm}$ in thickness, very similar to that predicted from the fitting of the ellipsometry data at the end of the growth phase in Fig. $3(160 \mathrm{~nm})$.

\subsubsection{Degradation of PANI films}

Fig. 3 shows that the $\Delta$ and $\Psi$ values are different at the start of the degradation experiment (point c), i.e. before the first period of holding at $1.0 \mathrm{~V}$, from those immediately after growth (point b). It should be noted that the PANI-coated electrode was removed from the aniline-containing solution immediately after growth, rinsed with triply de-ionized and distilled water, and stored in air overnight. The degradation experiments were not commenced until the following day in a fresh $1 \mathrm{~mol}^{1^{-1}}$ sulfuric acid solution. This change in the ellipsometric properties of PANI with time of storage and with a change in the solution has also been reported by Greef et al. [28].

The model yielding the best fit to the degradation data shows that the PANI film becomes thinner with time of holding at $1.0 \mathrm{~V}$, consistent with its expected dissolution [15-24]. The fact that the $n$ value of the film (1.52) is lower than during growth (1.58), after the first 6 min of holding at $1.0 \mathrm{~V}$ (point $\mathbf{c}$ to $\mathbf{x}_{\mathrm{d}}$ on the degradation curve of Fig. 3), indicates that the PANI film becomes more porous with the duration time of dissolution, probably also leading to an increasing extent of surface roughness.

The TEM analysis, carried out in parallel with the ellipsometry experiment, supports the suggested development of porosity during the anodic degradation of PANI films. Fig. 6 ( $a$ and $b$ ) show cross-sectional TEM 


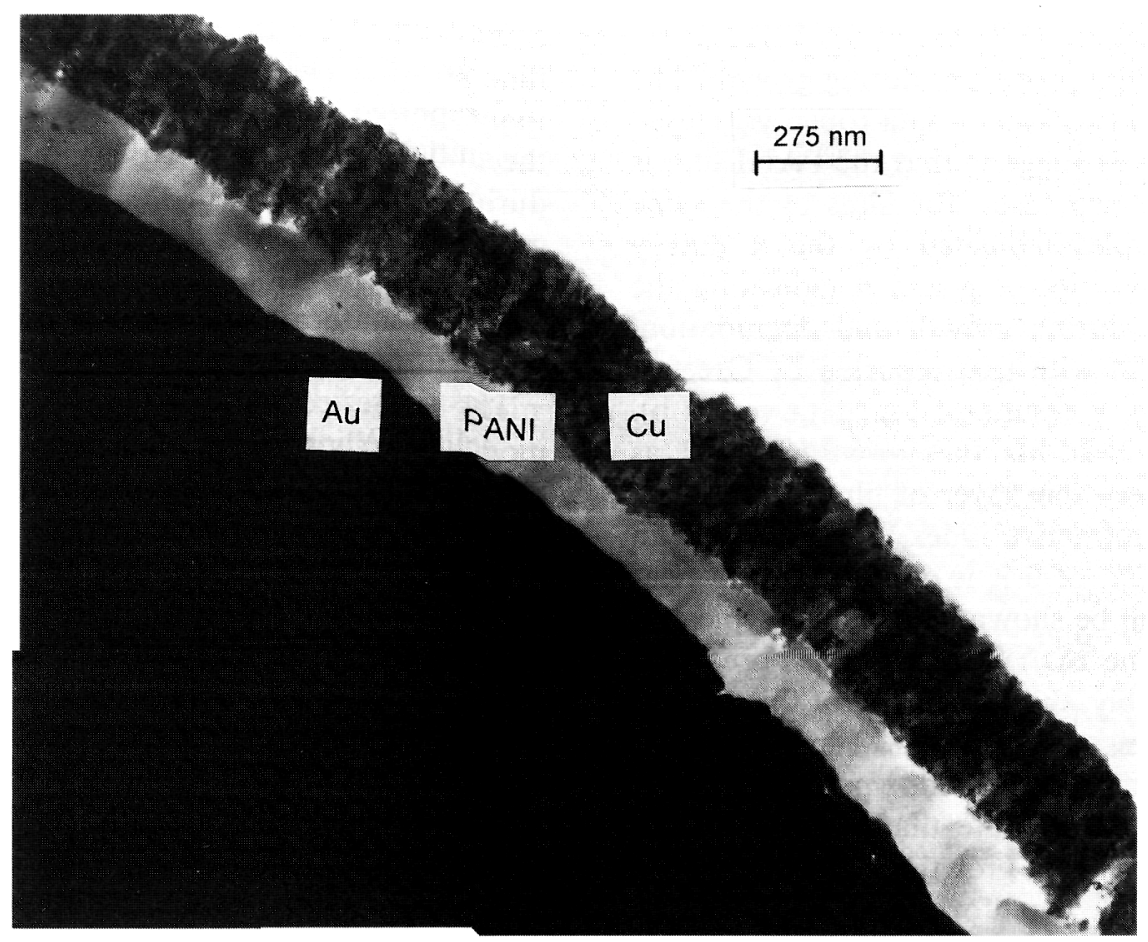

Fig. 5. Typical TEM cross-sectional view of a PANI film, for med electrochemically on a Au foil, using similar conditions as in ellipsometry experiments. The PANI film (light area, $160 \pm 10 \mathrm{~nm}$ thick) is sandwiched between the Au substrate and an overlayer of sputtered Cu.

views of a partially degraded PANI film. This particular film was removed from solution when ca. $30 \%$ of its original charge had been lost through time spent at 1.0 $\mathrm{V}$, which is equivalent to point $\mathbf{x}_{\mathbf{d}}(\mathrm{ca} .50 \mathrm{~nm}$ ) in the degradation curve of Fig. 3. Fig. 6 (a) shows that the film is uniform in nature and that its thickness of $75 \pm 5$ $\mathrm{nm}$ is ca. $70 \%$ of its expected original thickness $(105$ $\mathrm{nm}$ ). A greater porosity, particularly at the outer film surface, is seen by the fuzziness of the resolution at this interface and by the fact that the sputtered $\mathrm{Cu} \mathrm{O}_{\text {ver- }}$ layer appears to penetrate into the PANI film in some locations (Fig. 6b). It is also notable that no evidence can be seen for a loss of adhesion of this partially degraded film from the $\mathrm{Au}$ substrate, showing that delamination is not a factor which needs to be considered, consistent with the predictions from the ellipsometry data.

Fig. 3 shows also that over this same period of time of degradation, i.e. from point $\mathbf{c}$ to $\mathbf{x}_{\mathbf{d}}$ in the degr ${ }^{\mathrm{a} d a-}$ tion curve, the $k$ value $(0.014)$ is significantly larger than it was during the latter stages of PANI film growth (0.0031). A higher $k$ value would be consistent with the change of film color seen visually in the early times of film degradation, from $\mathbf{c}$ to $\mathbf{x}_{\mathbf{d}}$. It is not ${ }_{\mathrm{able}}$ that, during film growth, the highest $k$ value was see $n$ the first three to five cycles of potential, when the BQ/HQ redox peaks are prevalent (Fig. 1a). In fact, an inspection of Fig. 1 (b) shows that, after the first mi hute of film degradation at $1 \mathrm{~V}$, the BQ redox peaks, centeres at $\Omega .75-\Omega .8 \mathrm{~V}$, are a dominant feature $\Omega f$ the $C V$ response for the partially degraded film. These peaks do not increase or decrease significantly in size, in contrast to the large reduction in size of the main PANI redox peaks, until ca. $25 \%$ of the film has been removed. It is therefore reasonable to suggest that the higher value of $k$ in the early stages of film degradation reflects the presence of a $\mathrm{BQ} / \mathrm{HQ}$ altered polymer film in a predominantly PANI film matrix.

It should be pointed out that the degradation curve from point $\mathbf{c}$ to $\mathbf{x}_{\mathbf{d}}$ in Fig. 3 was modeled by keeping the properties of the as-grown inner layers of the PANI film constant, i.e. the inner portion of the film, consisting of the $2 \mathrm{~nm}(n=1.5, k=0.1)$ and the $40 \mathrm{~nm}$ ( $n=1.65, k=0.005$ ) layers, remained unchanged during the initial period of film degradation. This means that the increase in porosity and the formation of a $\mathrm{BQ} / \mathrm{HQ}$ altered polymer film, observed during the early stages of film degradation, occur mainly in the outer portion of the film.

From point $\mathbf{x}_{\mathbf{d}}$ to $\mathbf{z}_{\mathbf{d}}$ on the degradation curve of Fig. 3 , the ellipsometry data shows that film dissolution continues to occur with time spent at $1.0 \mathrm{~V}$. The properties of the inner layer are now clearly affected, as seen by the fact that the $n$ and $k$ values of the two inner layers had to be changed in order to fit the calculated $\Delta$ and $\Psi$ values to the experimental data. The relatively lower $n$ and higher $k$ values, compared to those during PANI film growth, indicate that the film porosity continues to increase and that more of the highly colored $B Q / H Q$ species are being formed. Correlating with this 


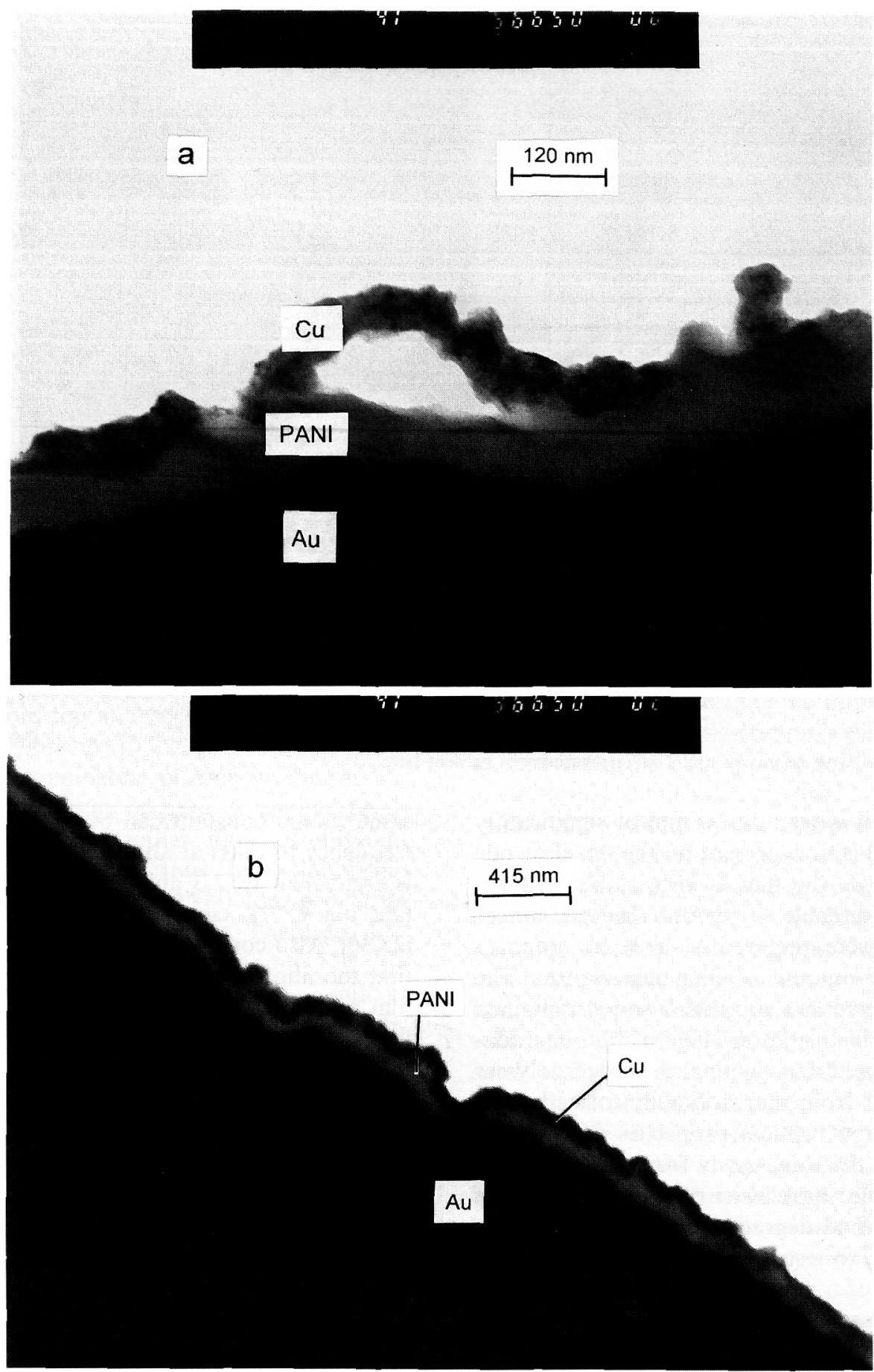

Fig. 6. TEM cross-sectional view of a partially degraded PANI film $(75 \pm 5 \mathrm{~nm})$, after ca. $30 \%$ of original film charge has been lost, at two different magnifications: (a) is ca. 3.5 times higher magnification versus (b). The original film thickness was predicted to be ca. $105 \mathrm{~nm}$. Fig. $6 \mathrm{~b}$ shows $\mathrm{Cu}$ overlayer partially penetrates PANI film in some locations.

stage, the CVs in Fig. 1 (b) show that now, both the PANI redox peaks and the peaks associated with the $\mathrm{BQ}$ or other polymeric degradation products, begin to decrease in size. A TEM cross-sectional analysis of a film at this stage of degradation (Fig. 7) shows the presence of two film layers, a relatively uniform inner layer (ca. $45 \mathrm{~nm}$ in thickness) and the remnants of a porous, outer layer. The PANI film shown in Fig. 7 has been degraded until only ca. $45 \%$ of its $\mathrm{CV}$ charge density has been lost. The inner layer is seen to be still adhering very well to the substrate.

When ca. $65 \%$ of the film has been lost by anodic dissolution/degradation (point $\mathbf{z}_{\mathbf{d}}$ in the degradation curve of Fig. 3), i.e. when the outer, porous PANI layer 


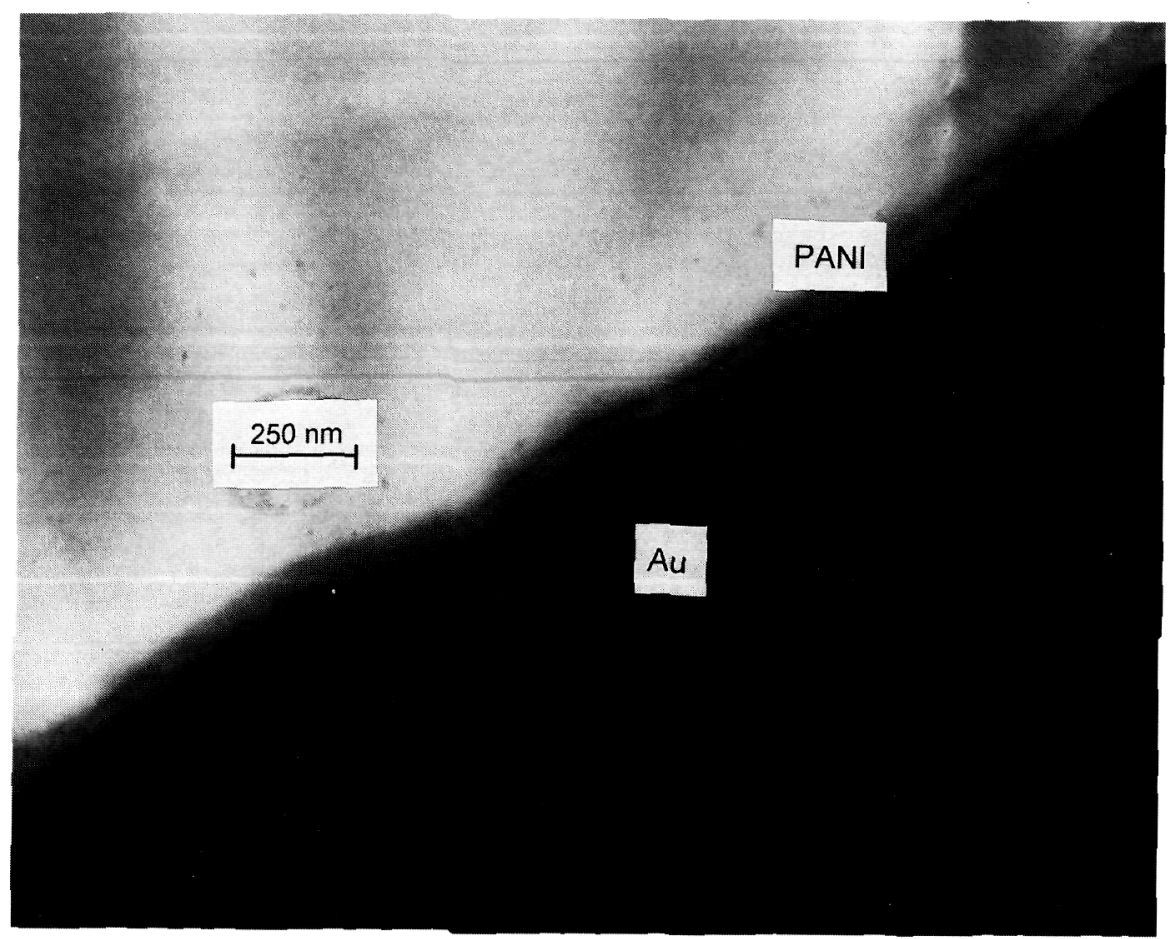

Fig. 7. TEM cross-sectional view of a degraded PANI film (ca. $45 \pm 5 \mathrm{~nm}$ ), after ca. $45 \%$ of original film charge has been removed. The original film thickness was predicted to be ca. $85 \mathrm{~nm}$. No $\mathrm{Cu}$ overlayer is present here.

has been dissolved, it is seen that a film of significantly higher $k$ value (0.05) is now present on the Au electrode surface. At this stage, the film is very stable and becomes increasingly difficult to remove. Indeed, longer times $(5-10 \mathrm{~min})$ were required at $1 \mathrm{~V}$ in order to induce even a small amount of film degradation. The $\mathrm{CV}$ charge barely decreased and the $\Psi$ and $\Delta$ remained essentially constant in this latter stage of film degradation. It should be noted that the final degraded polymer film is very different from that originally formed, as is shown by the different optical properties of the final polymer film versus the thin, newly formed PANI film. Furthermore, it would have been of interest to obtain an analysis of this final degraded film for its nitrogen and oxygen content, to test the hypothesis made above of the development of a new PANI/quinoneimine polymeric phase with time of anodic degradation. However, this was not possible with our existing instrumentation.

\section{3. $Q C M B$ measurements during the PANI film degradation process}

\subsubsection{Total film mass changes}

Fig. 8 shows that the total mass gain of the Aucoated crystal (measured at $0.0 \mathrm{~V}$ ) is linear with increasing charge density measured in slow sweep CVs between 0 and $0.9 \mathrm{~V}$ during PANI deposition. The fact that the plot goes through the origin in Fig. 8 is an indication that the PANI film is not perturbing the piezoelectric response of the quartz crystal, and that the same crystal constant can be used in the conversion of frequency to mass at all film thicknesses [18]. The slope is equivalent to 2.3 aniline groups per electron passed $\left(2.2 \mathrm{mg} \mathrm{C}^{-1}\right)$, close to the literature value of $\mathrm{ca} .2$ $[25,36]$. Also consistent with the literature, Fig. 9 shows that the film density, obtained from a plot of the total film mass versus the film thickness, obtained from the fitted ellipsometric data during PANI growth (Fig. 3), is ca. $1.0 \mathrm{~g} \mathrm{~cm}^{-3}$ overall. Gottesfeld et al. obtained a density of $1.36 \mathrm{~g} \mathrm{~cm}^{-3}$ for PANI films formed using

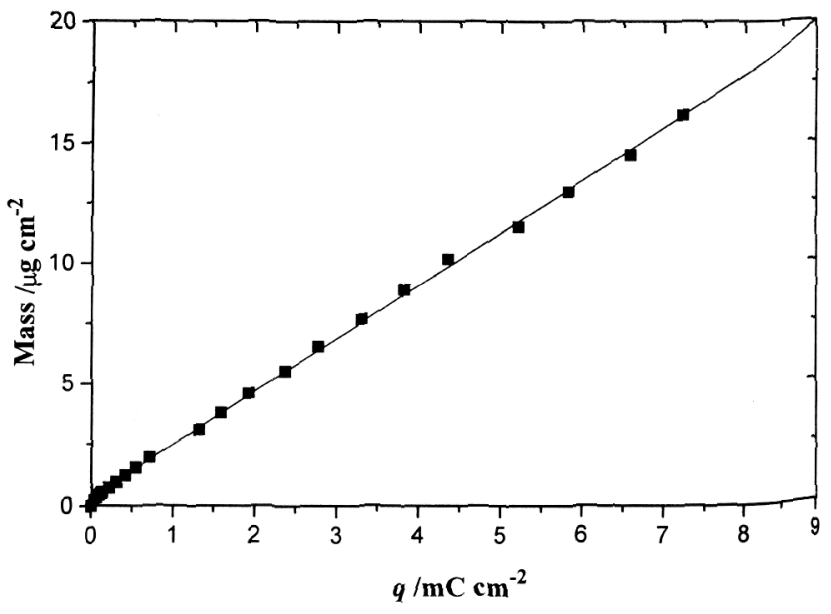

Fig. 8. Total film mass (measured at $0.0 \mathrm{~V}$ ) versus anodic charge density passed between 0.0 and $0.9 \mathrm{~V}$ during PANI growth on a $\mathrm{Au} /$ quartz crystal substrate in $0.1 \mathrm{~mol} 1^{-1}$ aniline $+1 \mathrm{~mol} \mathrm{I}^{-1}$ $\mathrm{H}_{2} \mathrm{SO}_{4}$. Slope is $2.2 \mathrm{mg} \mathrm{C}^{-1}$. 


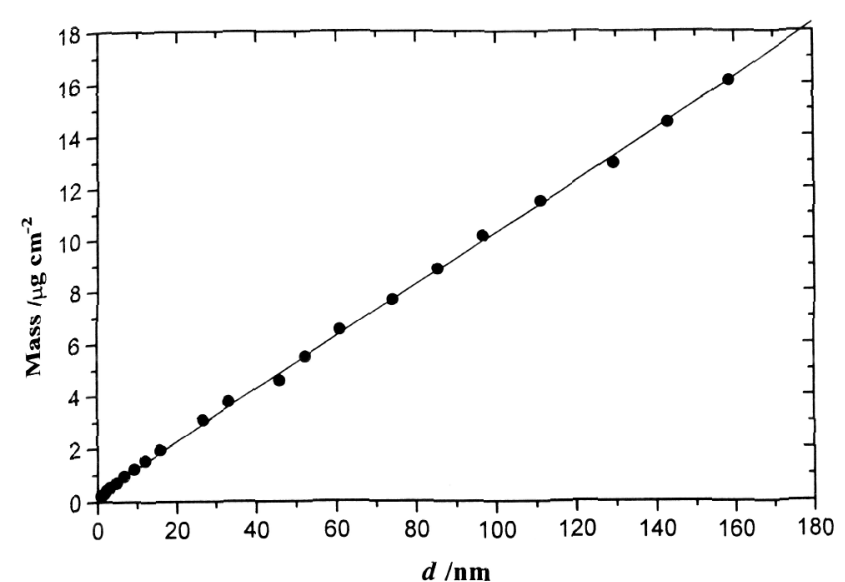

Fig. 9. Total film mass (measured at $0.0 \mathrm{~V}$ ) versus the film thickness, obtained from the fitted ellipsometric data (Fig. 3), during PANI growth on a $\mathrm{Au} \mid$ quartz crystal substrate in $0.1 \mathrm{~mol} \mathrm{l}^{-1}$ aniline +1 mol $1^{-1} \mathrm{H}_{2} \mathrm{SO}_{4}$. Film density is $1.0 \mathrm{~g} \mathrm{~cm}^{-3}$ from slope.

constant current methods, and values in the range of $1-1.5 \mathrm{~g} \mathrm{~cm}^{-3}$ for films formed at constant potential [31]. The similarity in the literature film densities to our value can be taken as support for the model of film growth obtained from the analysis of the ellipsometry data in the present work.

Fig. 10 shows the analogous plot as in Fig. 8, but during the film degradation process. A similar result has also been obtained for other films of different thicknesses in this study and thus the relationship shown is a true depiction of the PANI film properties during the anodic degradation process. There are several important points to be stressed about Fig. 10. First, it is significant that during the first $3 \mathrm{~min}$ of holding at $1 \mathrm{~V}$, the total film charge diminishes by almost $20 \%$ (in agreement with the ellipsometry results), while the film mass change is significantly less (ca. 7\%). This indicates that some of the retained PANI film

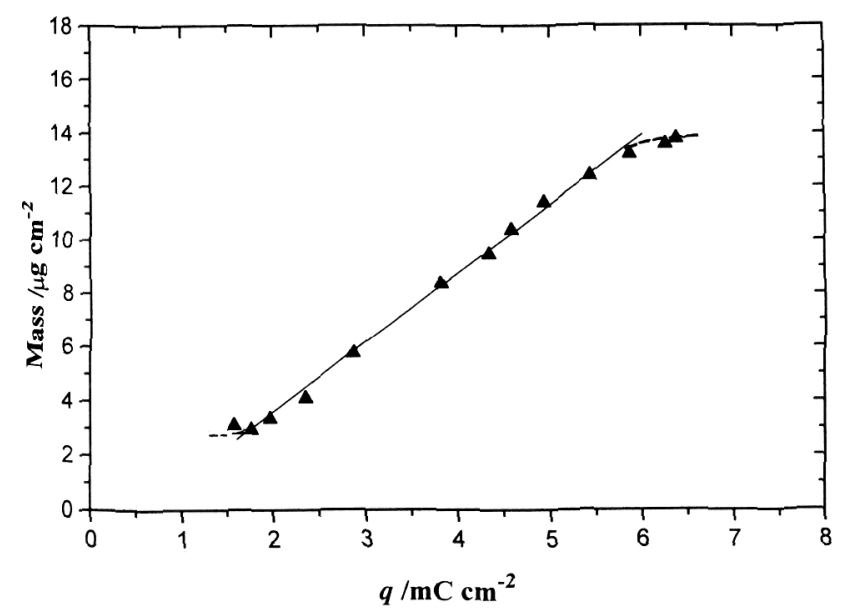

Fig. 10. Total film mass loss (measured at $0.0 \mathrm{~V}$ ) versus the anodic charge density measured between 0.0 and $0.9 \mathrm{~V}$ during PANI degradation on a $\mathrm{Au} \mid$ quartz crystal substrate in $1 \mathrm{~mol}^{-1} \mathrm{H}_{2} \mathrm{SO}_{4}$. Slope of the linear portion of the curve is $2.6 \mathrm{mg} \mathrm{C}^{-1}$.

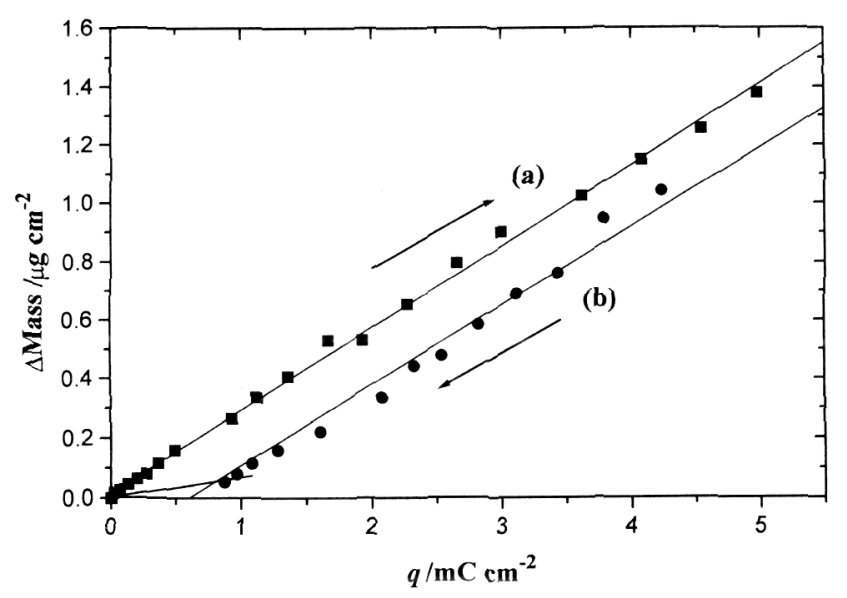

Fig. 11. Mass change versus the anodic charge density passed during cycling between 0.0 and $0.6 \mathrm{~V}$ during PANI (a) growth in $0.1 \mathrm{~mol}^{-1}$ aniline $+1 \mathrm{~mol} \mathrm{1}^{-1} \mathrm{H}_{2} \mathrm{SO}_{4}(\boldsymbol{\square})$ and (b) degradation in $1 \mathrm{~mol} \mathrm{l}^{-1}$ $\mathrm{H}_{2} \mathrm{SO}_{4}(\bullet)$.

becomes either electrically isolated or electrochemically non-reactive during this early phase of the degradation process. It is possible that the immediate development of $\mathrm{BQ} / \mathrm{HQ}$ sites during the early phase of degradation, as seen from the ellipsometry results, is related to this anomalously small mass change.

This is followed by the uniform dissolution of the film, during which time the relationship between the loss of mass and of charge is linear. The slope of this part of the plot in Fig. 10 yields a ratio of 2.7 aniline units per electron, showing that the partially degraded film is less active, overall, than it was during its formation, since more aniline is now required to generate the same amount of charge. This is consistent with the small mass loss but substantial loss of charge in the early phase of PANI degradation, discussed above.

Finally, it is of interest that when the degradation process reaches the stage at which further charge loss becomes difficult (when the layer of $k=0.05$ is reached and the ellipsometric data remain essentially constant), the charge decreases only very slowly, while the mass of the film remains relatively constant. This demonstrates that, even at this stage of film degradation, more of the remaining film is becoming electrically isolated from the gold substrate, and/or that the degradation products being formed are insoluble, but electrochemically inactive. Consistent with this, Stilwell et al. have suggested that the remaining PANI film residue forms via crosslinking reactions, yielding unreactive residues after anodic degradation [20]. It should also be noted that the QCMB data of Fig. 10 do not show any evidence which would indicate the occurrence of film delamination with time spent at $1 \mathrm{~V}$.

\subsubsection{Mass changes during film oxidation/reduction}

The mass change associated with the ion and solvent 
injection/expulsion processes which occur during charge compensation of PANI as the potential is scanned positively through its redox peak $(0-0.6 \mathrm{~V})$ was also examined (Fig. 11), both for freshly grown films and for films at various stages of degradation. During film growth, the slope of the plot is linear, averaging at $29 \mathrm{~g}$ $\mathrm{mol}^{-1}$ of electrons passed over this potential range (curve a, Fig. 11). This value has been interpreted previously as reflecting a mixture of proton expulsion, bisulfate injection and water injection during film oxidation up to $0.6 \mathrm{~V}$, and vice versa during reduction [37]. For a film which has been anodically degraded in stages, Fig. 11 (curve b) shows that a similar slope of 28 $\mathrm{g} \mathrm{mol}^{-1}$ of electrons is seen for the majority of the degradation period. When the very stable, fully degraded layer of film is reached after long times of degradation, the mass to charge ratio becomes very small, perhaps indicative of only protonic charge compensation, which would yield a negligible predicted mass change $\left(1 \mathrm{~g} \mathrm{~mol}^{-1}\right.$ of electrons). This change in the charge compensation mechanism within the $0-0.6$ $\mathrm{V}$ potential range to only protonic may be related to the movement of the PANI redox peak to higher potentials (Fig. 1b), as negligible mass changes are usually observed at the onset of PANI oxidation.

Fig. 12 shows the analogous set of data to that in Fig. 11, but for the range of potential between 0.6 and $0.9 \mathrm{~V}$. This analysis was done in order to examine the nature of the charge compensation mechanism in the BQ peaks, especially as the film becomes degraded. During film growth, Fig. 12 (curve a) shows that the mass to charge ratio in the early stages, when the BQ peak is present (Fig. 1 (a), first 60 cycles), is ca. $59 \mathrm{~g}$ $\mathrm{mol}^{-1}$ of electrons. This ratio would be consistent with the majority of the charge compensating ions being the bisulfate ion. As the film thickens, and the main contri-

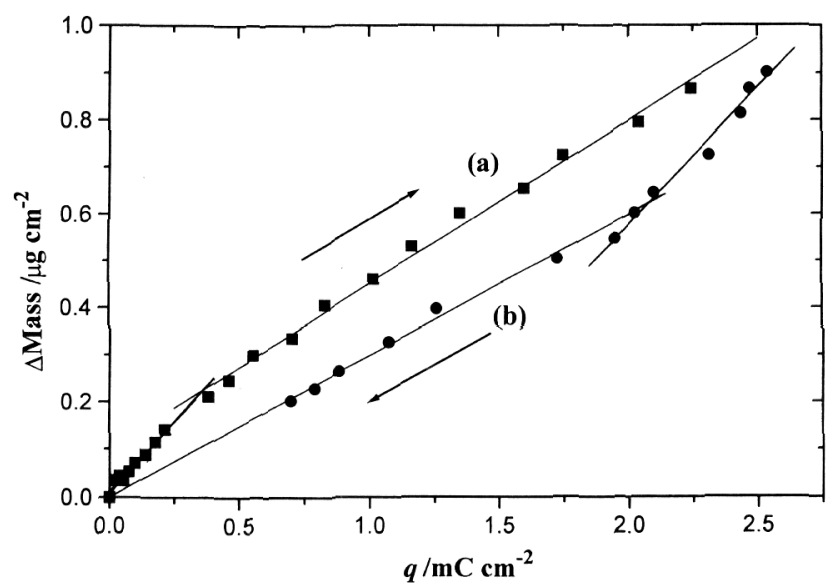

Fig. 12. Mass change versus the anodic charge density passed during cycling between 0.6 and $0.9 \mathrm{~V}$ during PANI (a) growth $0.1 \mathrm{~mol} 1^{-1}$ aniline $+1 \mathrm{~mol} \mathrm{1}^{-1} \mathrm{H}_{2} \mathrm{SO}_{4}(\boldsymbol{\square})$ and (b) degradation $1 \mathrm{~mol} \mathrm{l}^{-1}$ $\mathrm{H}_{2} \mathrm{SO}_{4}(\bullet)$. bution to the charge in this range of potential is the completion of the principal PANI redox process $(\mathrm{Fig}$. 1a), rather than the $B Q / H Q$ reaction, the ratio de. creases to 36 , more similar to the ratio of $29 \mathrm{~g} \mathrm{~mol}^{-1}$ seen in the PANI peak potential range in Fig. 11 (curve a).

Fig. 12 (curve b) is the analogous plot to Fig. 12 (curve a), but during film degradation. In the early stages, when the BQ redox peaks are clearly seen (Fig. $1 \mathrm{~b})$, the ratio is seen to be $60 \mathrm{~g} \mathrm{~mol}^{-1}$ of electrons, very similar to the higher slope of 59 seen at early growth times in Fig. 12 (curve a), also when the $\mathrm{BQ}$ peaks were the predominant feature in this potential range. The higher mass to charge ratio of 60 seen at the commencement of film degradation indicates that the anodic charge compensation process involves primarily the injection of bisulfate ion, rather than the expulsion of hydrated protons. This may suggest that some hydrated protons remain in the film, facilitating the degradation reaction (hydrolysis) of PANI films. Fig. 12 (curve b) shows that the slope decreases to $31 \mathrm{~g} \mathrm{~mol}^{-1}$ of electrons for the remainder of the degradation process. This slope is most similar to that seen in the PANI redox peaks during growth and much of the degradation process.

Taken together, Figs. 11 and 12 suggest that when the $\mathrm{BQ}$ peaks are the dominant feature in the range, $0.6-0.9 \mathrm{~V}$, the mass to charge ratio is ca. 60 grams per moles of electrons, both during film growth and degradation. After longer times at $1 \mathrm{~V}$, the ratio of 30 grams per moles of electrons, characteristic of PANI, is seen at all potentials. When the film becomes resistant to further degradation and the PANI redox peaks begin to move anodically, merging with the BQ peaks, the mass to charge ratio is negligible in the $0-0.6 \mathrm{~V}$ range, while a ratio of $30 \mathrm{~g} \mathrm{~mol}^{-1}$ of electrons is seen in the range $0.6-0.9 \mathrm{~V}$. These results show that, while it is clear that the overall characteristics of the residues are quite different from the original PANI film, the charge compensation mechanism of the remaining film reflects only the presence of PANI.

\section{Summary}

The main objective of this work has been to examine the nature of the polyaniline (PANI) anodic degradation process, primarily to establish the structural/morphological and electrochemical properties of the polymer film with extent of degradation. PANI films were formed to a range of thicknesses up to $160 \mathrm{~nm}$, by potential cycling between 0.0 and $0.95 \mathrm{~V}$, on Au electrodes in sulfuric acid solutions. In the first few potential cycles, a pronounced anodic peak at ca. $0.75 \mathrm{~V}$, attributed in the prior literature to be the redox potential of benzoquinone (BQ), is the dominant feature. 
During this early phase of growth, ellipsometric studies show that a film of somewhat higher absorptivity than the reduced, transparent PANI forms. With further growth, the PANI redox peak begins to dominate the $\mathrm{CV}$ response and the ellipsometric data show that a compact and then a more porous overlayer grows at a rate which increases with time under these conditions. The final film thickness predicted from the ellipsometric data fitting is in agreement with that established from TEM cross-sectional analysis. In situ mass measurements (QCMB technique) reveal that the film mass increases linearly with the CV charge density, and that the charge compensation stoichiometry in the potential range of the PANI redox peaks is different from that in the $B Q$ peaks.

PANI films were anodically degraded by holding the potential at $1 \mathrm{~V}$ in sulfuric acid solutions for various periods of time. Ellipsometry shows the immediate formation of colored species in the film, perhaps containing benzoquinoid endgroups. The concurrent $\mathrm{CV}$ response shows that the peak centered at ca. $0.75 \mathrm{~V}$ is now the dominant feature, suggesting that degradation products, perhaps mainly $\mathrm{BQ}$, are deposited within the film. In parallel with this, the bulk of the film dissolves uniformly, creating a greater porosity. Ultimately, a thin film of very different properties remains on the surface. This model of film degradation has been supported by TEM studies of cross-sectional samples of PANI films, which also showed the development of increasing film porosity and film thinning, as well as the ultimate formation of a thin layer of film that is very resistant to further dissolution/degradation.

Concurrent in situ mass measurements, carried out during PANI degradation, suggest that in the early stages, some regions of the film become electrically inactive. However, the charge compensation mechanism in the PANI redox peaks remains the same as during film growth until only the final, relatively inactive film material is left on the Au surface. At higher potentials, it appears that the charge compensation process during the early stages of degradation is similar to that seen when the BQ redox peaks are present at the beginning of film growth. In the latter stages of film degradation, the mass data show that a large quantity of the film is inactive. Furthermore, the PANI redox peak begins to move positively, almost merging with the $\mathrm{BQ}$ redox peaks, so that charge compensation of PANI occurs at more positive potentials. Therefore, only protonic charge compensation is seen between 0 and $0.6 \mathrm{~V}$, while a charge compensation stoichiometry of $30 \mathrm{~g} \mathrm{~mol}^{-1}$ of electrons, typical for PANI redox peaks, is observed at higher potentials. While it is clear that an altered polymer film is now present, the charge compensation mechanism indicates that only those parts of the film that have PANI characteristics are electrochemically active. Finally, there is no evidence to support the contention that the PANI film has been undermined.

\section{Acknowledgements}

The authors are grateful to the Natural Sciences and Engineering Research Council of Canada (NSERC) for their overall support of this work. H.N. Dinh also acknowledges scholarship support from NSERC, IODE, Alberta Heritage Foundation, A.S.M. International 'Calgary Chapter', and the University of Calgary. The authors also wish to thank Dr. P. Vanýsek, NIU, for useful discussions regarding QCMB experiments.

\section{References}

[1] P. Fiordiponti, G. Pistoia, Electrochim. Acta 34 (1989) 215.

[2] J.C. Lacroix, K.K. Kanazawa, A. Diaz, J. Electrochem. Soc. 136 (1989) 1308.

[3] T. Kobayashi, H. Yaneyama, H. Tamura, J. Electroanal. Chem. 161 (1984) 419.

[4] L.M. Goldenberg, M.C. Petty, A.P. Monkman, J. Electrochem. Soc. 141 (1994) 1573.

[5] A.G. MacDiarmid, S.-L. Mu, N.L. Somasiri, W. Wu, Mol. Cryst. Liq. Cryst. 121 (1985) 187.

[6] C. Barbero, M.C. Miras, B. Schryder, O. Haas, R. Kötz, J. Mater. Chem. 4 (1994) 1775.

[7] G. Mengoli, M.M. Musiani, G. Zotti, S. Valcher, J. Electroanal. Chem. 202 (1986) 217.

[8] L. Doubova, G. Mengoli, M.M. Musiani, S. Valcher, Electrochim. Acta 34 (1989) 337.

[9] G. Mengoli, M.T. Munari, P. Bianco, M.M. Musiani, J. Appl. Polym. Sci. 26 (1981) 4247.

[10] G. Mengoli, M.M. Musiani, B. Pelli, E. Vecchi, J. Appl. Polym. Sci. 28 (1983) 1125.

[11] S. Sathiyanarayanan, S.K. Dhawan, D.C. Trivedi, K. Balakrishnan, Corros. Sci. 33 (1992) 1831.

[12] S. Sathiyanarayanan, K. Balakrishnan, S.K. Dhawan, D.C. Trivedi, Corros. Sci. 39 (1994) 831.

[13] G. Troch-Nagels, R. Winand, A. Weymeersch, L. Renard, J. Appl. Electrochem. 22 (1992) 756.

[14] B. Wessling, Adv. Mater. 6 (1994) 226.

[15] T. Kobayashi, H. Yoneyama, H. Tamura, J. Electroanal. Chem. 177 (1984) 293.

[16] D.E. Stilwell, S-M Park, J. Electrochem. Soc. 135 (1988) 2491.

[17] D. Orata, A. Matheka, B. Munge, Macromol. Chem. Phys. 195 (1994) 3003.

[18] D. Orata, D.A. Buttry, J. Am. Chem. Soc. 109 (1987) 3574.

[19] C. Barbero, M.C. Miras, O. Haas, R. Kötz, J. Electroanal. Chem. 310 (1991) 437.

[20] D.E. Stilwell, S.-M. Park, J Electrochem. Soc. 135 (1988) 2497.

[21] V. Tsakova, A. Milchev, Electrochim. Acta 36 (1991) 1579.

[22] A.Q. Zhang, C.Q. Cui, J.Y. Lee, Synt. Met. 72 (1995) 217.

[23] R.L. Hand, R.F. Nelson, J. Am. Chem. Soc. 96 (1974) 850.

[24] R.L. Hand, R.F. Nelson, J. Electrochem. Soc. 125 (1978) 1059.

[25] C.Q. Cui, L.H. Ong, T.C. Tan, J.Y. Lee, Electrochim. Acta 38 (1993) 1395.

[26] F.L. McCrackin, NBS, Technical Note 479 (1969).

[27] G. Sauerbrey, Z. Phys. 155 (1955) 206.

[28] R. Greef, M. Kalaji, L.M. Peter, Faraday Discuss. Chem. Soc. 88 (1989) 277.

[29] S. Gottesfeld, A. Redondo, S.W. Feldberg, Electrochem. Soc Extended Abstracts, San Diego, CA, Abstract 507 (1986) 759.

[30] A. Redondo, E.A. Ticianelli, S. Gottesfeld, Mol. Cryst. Liq Cryst. 160 (1988) 185. 
[31] J. Rishpon, A. Redondo, C. Deroin, S. Gottesfeld, J. Electroanal. Chem. 294 (1990) 73.

[32] E. Sabatani, A. Redondo, J. Rishpon, A. Rudge, I. Rubinstein, S. Gottesfeld, J. Chem. Soc. Faraday Trans. 89 (1993) 287.

[33] S. Gottesfeld, A. Redondo, S.W. Feldberg, J. Electrochem. Soc. 134 (1987) 271.
[34] C. Barbero, R. Kötz, J. Electrochem. Soc. 141 (1994) 859.

[35] B.J. Johnson, S-M Park, J. Electrochem. Soc. 143 (1996) 1277.

[36] F. Rourke, J.A. Crayston, J. Chem. Soc. Faraday Trans. 89 (1993) 295

[37] H.N. Dinh, V.I. Birss, J. Electroanal. Chem. 443 (1998) 63. 